\title{
The Integrated Design for Micro - environment Monitoring System of Showcase in Museum
}

\author{
Chan Dong ${ }^{1}$, Yang Zhang ${ }^{2}$, Jingjie $\mathrm{Li}^{1}$ and Hui Zhang ${ }^{2, *}$ \\ ${ }^{1}$ Department of Instrument Intelligent Manufacturing Technology, Hefei University of Technology, Hefei, China \\ ${ }^{2}$ Department of Instrument Science and electronic engineering, Hefei University of Technology, Hefei, China \\ Corresponding Email: zhanghui@hfut.edu.cn
}

\begin{abstract}
In order to improve the current environment quality of cultural relics in museum and make them preserved for a long time, the paper proposes the integrated design for micro environment monitoring system of showcase in museum, the system mainly monitors the micro-environment of heritage, such as ultraviolet, light, formaldehyde, $\mathrm{CO}_{2}, \mathrm{TVOC}$, PM2.5, choosing cotex-M3 ARM microcontroller STM32F103ZET6 as the control core. Based on the concept of integration, the system integrates the single air monitoring instruments. The design of circuit mainly includes the process of digital power supply and analog power supply, the acquisition and processing of 5 analog signals from sensors, and the design of reserved interface. In the aspect of interaction, the serial port led module was uesd for the display and control, which can get rid of the control of PC and achieve the functions of environmental monitoring, environmental warning, environmental assessment, historical data query. The integrated design for Micro-environment of Showcase in Museum achieves a monitoring platform successfully which is easy for users' operation and access to display information conveniently. The advantages of the system are strong portability,low cost and short development cycle.
\end{abstract}

Keywords.STM32, micro-environmental monitoring, museum showcase, human-computer interaction.

\section{Introduction}

The 12th Five-Year plan for the development of the National Museum of Antiquities put forward that establishment of the support system of museum quality monitoring [1], environmental assessment and regulatory should be considered as a major research content. In recent years, the related industries are committed to the research of the micro-environmental monitoring of showcase [2]. As a symbol of national cultural soft power, the protection of cultural relics has always been a top priority in museum management, the air quality of the showcase is vital to the preservation of exhibits.

At present, the parameters monitored by the monitoring terminal on the market are relatively simple, which cannot achieve the all-round monitor. So the comprehensive micro-environmental monitoring system was designed for a showcase. This system integrates a plurality of various single-parameter air monitor, the monitoring of the four major polluting gases can be integrated, also include light and ultraviolet, the major four polluting gases include formaldehyde, $\mathrm{CO}_{2}, \mathrm{PM} 2.5$ and TVOC, which have bad effects on the exhibits [3]. Meanwhile, the system comes with a good interactive interface, which is convenient for the users to get the information of the current micro-environment and deal with it in time.

\section{Overview of museum micro- environment monitoring system}

The museum micro-environment monitoring system which is used to monitor the environment of cultural relics in the showcase. The purpose is to preserve the cultural relics in a safe and applicable condition of exhibition, in order to maintain the overall appearance of the showcase; the whole system is hid in the bottom of the showcase.

To comprehensively improve the level of environmental monitoring, control of showcase and storehouse. Technical Research Institute of Machinery Industry Instrument and Meter Industrial Technology Research Institute held a meeting which is about the standard for protective equipment of cultural relics. Including 28 standards of protection and safety requirements of cultural relics prevention equipment [4], evaluation methods, performance monitoring, intelligence terminal, multifunctional mobile showcase platform. For monitoring terminal. Online monitoring content should include 
temperature and humidity, formaldehyde, particulate matter (PM10 and PM2.5), light and ultraviolet light, carbon dioxide, volatile substances [5], etc. and the lowest performance index of the product is established.

According to this standard, The integrated design of six kinds of parameters was realized, which is including ultraviolet, light and illumination, formaldehyde, PM2.5, TVOC and $\mathrm{CO}_{2}$, considering the different adaptability of different cultural relics to the environment, the system can set the threshold, different cultural relics set different monitoring intervals.

In the design of the structure, the system adopts modular design, each module will be treated appropriately, in order to obtain higher precision. At the same time, the system comes with interactive interface, which have brought great convenience to the museum staff and visitors, the interactive interface focus on art design, strive to achieve consistent aesthetics with cultural relics.

\section{Hardware design of monitoring system}

\subsection{Composition of monitoring system}

Showcase micro-environment monitoring system block diagram was shown in Figure 1.

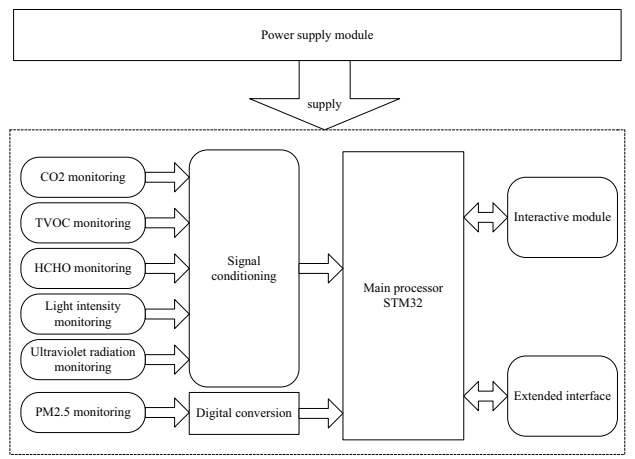

Fig. 1. Micro - environment monitoring system block diagram of showcase.

The system is based on the STM32F103ZET6 ARM cortex-M3 micro controller. The chip is rich in resources and fully meets the design requirements. That included power module, 5-way AD signal acquisition, signal conditioning, 1-way RS485 communication, interactive module, and reserved expansion interface.

The main power module provides the main processor, interactive modules, each sensor with their own required voltage.

$\mathrm{AD}$ signal conditioning mainly for the precision of the measurement requirements, a reasonable choice of internal AD or external $\mathrm{AD}$ for data collection [6].

Interactive module is the mainly based on 7-inch serial LCD and the main controller through RS232 communication to achieve a variety of interactive interface.

Taking into account higher air quality requirements of showcase micro environment, the resolution and precision of the air parameters must meet the requirements. Therefore, the system of the power module, the hardware design of the data acquisition part, the sensor output signal processing as well as the PCB has done the corresponding processing in order to obtain the higher measurement precision.

\subsection{Power supply module}

Stable and reliable power supply is an important part of system, at the same time; communication acquisition module requirements are relatively high not only on the system power supply stability but also anti-jamming performance. The system power supply block diagram was shown in Figure 2.

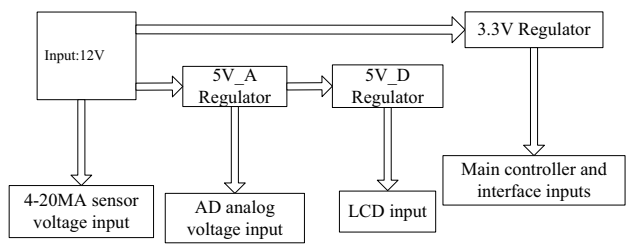

Fig. 2. Micro - environment monitoring system power management block diagram of showcase. 
Taking the influence of power circuit on signal transmission into account, the power isolation was done in the part of mutual interference, the $12 \mathrm{~V}$ DC power provide input for the light sensor, UV sensor. $12 \mathrm{~V}$ will be converted to $5 \mathrm{~V} \_\mathrm{A}$ through the three-terminal regulator LM2576. While the use of isolated DC-DC voltage module will convert $12 \mathrm{~V}$ to 5V_D, supply power for the LCD. And then through the corresponding filter for external AD power supply. the 5V_D converted to DC 3.3V voltage through ASM1117-3.3 [7], provides voltage input for the main controller, interface, and so on.

In addition, the analog power and digital power through the $0 \Omega$ resistor to connected GND and 3.3V respectively. Loop current can be suppressed, thereby suppressing the high-frequency noise of the digital ground [8]. When the PCB is covered with copper, separate the AD part from the rest that can reduce the interference of the circuit to AD.

\subsection{Data acquisition module}

The quality of the system was affected directly by Data collection accuracy. Therefore, In order to improve the accuracy of the monitoring system, the appropriate treatment was used for different types of sensors, also can meet the requirements for the indicators of the monitoring terminal, which was promulgated in the "cultural relics protection equipment standard assembly (a)" in 2016.

In the signal processing, light illumination sensor and ultraviolet sensor, which have a larger output current, the standard provides the measurement accuracy of the two sensor, respectively $10 \%$ and $5 \%$, so the internal AD was choosed to meet the measurement requirements directly.

According to T / WWXT0015-2015 standard [9],the measurement range and accuracy of $\mathrm{CO}_{2}$ monitoring terminal were $\{2000 \mathrm{ppm}, \pm(60 \mathrm{ppm} \pm 3 \%$ full range $)\}$, the model of $\mathrm{CO}_{2}$ sensor is COZIR-A2000 in the system, the range of measurement is $2000 \mathrm{ppm}$, so that meet the standard requirements fully. The output voltage of $\mathrm{CO}_{2}$ sensor was related to the input voltage, when the input voltage is $3.4 \mathrm{~V}$, the output is $3.4 \mathrm{~V}, \mathrm{CO}_{2}$ measurement accuracy is higher, so external $\mathrm{AD}$ was used for data collection, but the circuit voltage divider was need at first, followed by AD processing.

The model of formaldehyde sensor is EC803, the measurement accuracy and measurement range are able to meet the $\mathrm{T} / \mathrm{WWXT} 0015-2015$ standards, the input voltage is $5 \mathrm{~V}$ and the output voltage is $0-2 \mathrm{~V}$, it is the analog voltage output, formaldehyde detection required the higher resolution, so external $\mathrm{AD}$ was used for data processing, TVOC and other sensors are also selected in accordance with standard requirements.

External AD hardware design of circuit was shown in figure 3, the (1) is external AD signal acquisition, and the (2) is condition circuit design.

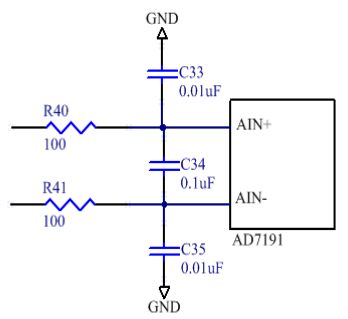

(1)

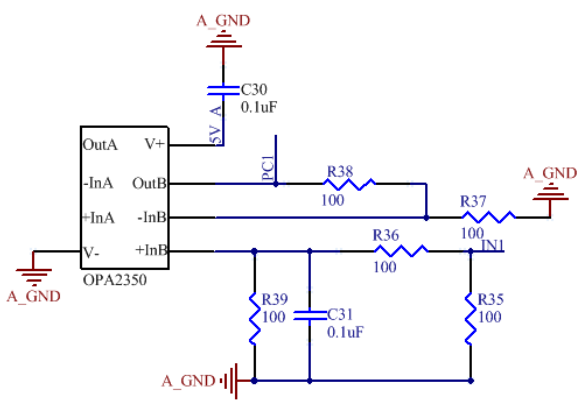

(2)

Fig. 3. Micro - environment monitoring Signal processing circuit design of showcase.

Since the input voltage of $\mathrm{CO}_{2}$ is $3.3 \mathrm{~V}$, it should be divided. In order to ensure the stability of the voltage, the voltage follower circuit is added after the separated voltage, and finally the $\mathrm{AD}$ processing, the accuracy of the Measurement is \pm (50ppm $\pm 2 \%$ full range), fully meet the requirements. 


\subsection{Interface design}

STM32F103VET6 master chip has very rich peripheral resources, 13 communication interfaces, clocked frequency up to 72Mhz. PM2.5 sensor uses serial to RS485 communication, and the rest are using ordinary interface input, a serial port to RS232 to communicate with the LCD screen, the interface information shown in Figure 4.

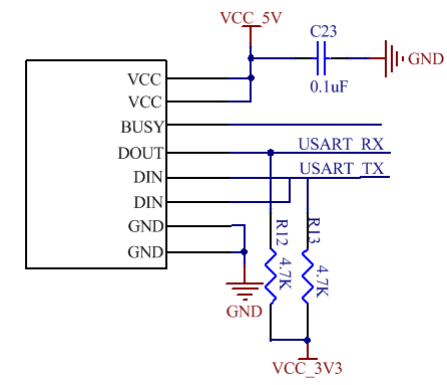

Fig. 4. Micro - environment monitoring LCD interface circuit design of showcase.

Take the expansion of the monitoring system to consideration. set aside a serial port for hardware and software debugging in the remaining resources, a RS485 communication interface is connected to the control, A USART interface is used for wireless communication.

\section{Software design of monitoring system}

Software design is based on hardware design in the system, including analog-digital conversion, real-time acquisition of 5-way sensor analog signal, a RS485 signal acquisition, processing, display, save and so on. The software design logic block diagram shown in Figure 5.

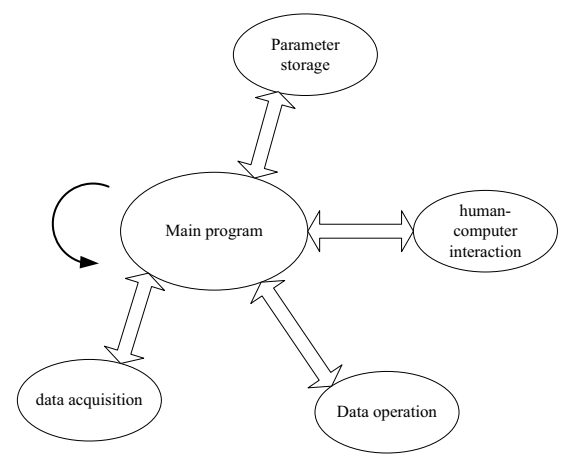

Fig. 5. Micro - environment monitoring system software design framework of showcase.

When the main program running, the timing full-period sampling of the system is opened, once enter a sample cycle, each sensor module to start data acquisition, the data of the collection will be operated, which includes the average, remove the gross error, the unit conversion, etc. The converted data into the human-computer interface for real-time display and update the data according to the set period.

Software Design of Interactive Interface.Flow chart of interactive software design shown in Figure 6. First, the whole initialization mainly includes the system equipment, the serial port, the clock, each variable and so on, second, go to the main interface of monitoring, all operations were responsed by users to user interface, the serial port will return the appropriate instructions, and then enter the communication subroutine and human-computer interaction subroutine respectively, the communication subprogram mainly completes the analysis to the serial port instruction, finally, sub-menu interface conversion operation was completed by human-computer interaction subroutine. 


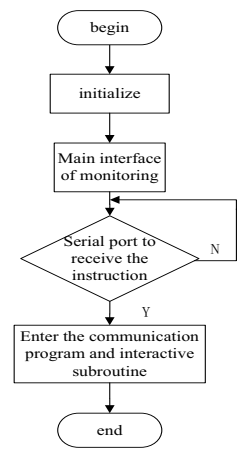

Fig. 6. Flow chart of interactive software design.

At the same time, the system can also be implemented the sensor state settings, threshold settings and other information on the parameters of storage, on the other hand, the host computer can send a time calibration command to calibrate the serial port screen real time.

\section{Realization of interactive interface}

At present, $80 \%$ of the micro-environmental monitoring terminal can not be separated from the PC-side control [10],that users can not access the current environment or related information directly.

Human computer interaction is gradually developing towards the direction of user experience. Users communicate with the system through interactive interface and a series of operations can be performed. A good human-computer interaction interface design can enhance the user's understanding of the system.

\subsection{On-line emulation debugging}

The user has defined the relevant items of the human-computer interaction, the simulation and online testing of human-computer interaction system can be completed without the support of the hardware, which is better than other display tools, using the serial port screen simulation software VisualTFT on the PC, virtual serial port screen. In the keil development environment, the virtual serial port contact with host computer through the virtual serial port, keil program debug simulation. The serial port screen will realize the function that corresponding communication record, also can monitor the host to the screen issued by the instruction, facilitate the process of analysis and debugging later.

Thus, the serial port screen is applied to the human-computer interaction system. It is a relatively independent unit, which can be synchronized with the development of instrument hardware, and will greatly shorten the project cycle, saving the project cost.

\subsection{Realization of Interactive Interface}

The main interface of environmental monitoring includes four secondary interfaces, there are environmental monitoring, environmental assessment, environmental warning, and historical data. Environmental monitoring to achieve the description of cultural relics information in current showcase, the display of monitoring data, the setting of working mode, as well as threshold settings. Set password-protected at the mode setting and threshold setting, non-staff members can not enter. Environmental assessment realizes the curve description of four kinds of detection gas. Environmental warning realizes the abnormal alarm mainly. Historical data realizes the current showcase address query and upload data query.

Take a second-level interface as an example, which is the environment warning communication display as shown in Figure 7. The second-level interface contains three three-level interfaces, including the early warning description, the time of warning, the type of warning. At the same time, the early warning description refers to the monitoring terminal mainly, it can tell the users which is abnormal at present. The warning time mainly refers to the time period that is viewed. The early warning type is classified according to the different abnormal conditions, mainly including the primary warning, secondary warning and high-risk warning. 


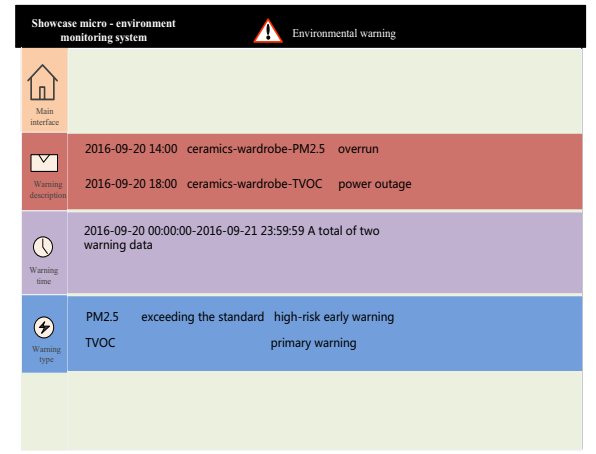

Fig. 7. Function diagram of Human computer interaction.

\section{Conclusion}

The micro environmental monitoring platform of showcase this paper has introduced, implant the concept of the integration into this design, and making the function of the products diversification. The products features can be combined freely, and the wide variety of type can meet the various needs of users. At the same time, for the visitors, the interactive interface design not only enhance the understanding of museum products, but also more conducive to museum staff to grasp the current environmental information timely, and then make effective management in time. The function of serial LCD online test also greatly shorten the development cycle and development costs were reduced.

From the perspective of product scalability, some functions will be increased, such as the environmental control, voice interaction, SMS alarm and so on. The monitoring system will has good development prospects.

\section{Acknowledgement}

This research was finacially supported by Key Project of Transformation and Industrialization of Scientific and Technological Achievements in Anhui Province.

\section{References}

1. Laiming $\mathrm{Wu}$, The seventh China Museum and related products and Technology Expo. 6(2016) 88-96.

2. Laiming $\mathrm{Wu}$, Promote Cultural Relic Prevention and Protection Ability by Developing Heritage Protection Equipment. 11(2015) 1134-1145.

3. Bo Zhang, Open heritage monitoring and early warning and protection decision-making management of the new model. 10 (2015) 266-276.

4. Joan Cahill, Nick Mc Donald, Human computer interaction methods for electronic flight bag envisionment and design. 9(2015)113-123.

5. Shengming WU. Smart home interactive terminal design and implementation. 14(2013).

6. Salaheldin Mohamed Ibrahim Edam, Key Technologies of Wireless Ad-hoc and Sensor Networks for Optimized Transmission Management in Disaster Situation. International Journal of Rock Mechanics \& Mining Sciences. 3(2014) 59-67.

7. Hui Tian, Design and Research of Data Center Micro - Environment Monitoring System Based on Zigbee. 5(2016) 207-214.

8. Xiaoting Li, Development of Serial LCD Based on Embedded System. 4(2014) 446-486.

9. Cultural relic protection equipment industry and application of collaborative work platform, Compilation of Standards for the Protection of Cultural Relics. (Cultural Relics Press, 2016) .

10. Gabor Aranyi, Paul Schaik, Modeling user experience with news websites. 4(2015) 59-66. 